\title{
Blowup for nonlinearly damped viscoelastic equations with logarithmic source and delay terms
}

\section{Sun-Hye Park ${ }^{1 *}$ D}

\section{"Correspondence:}

sh-park@pusan.ac.kr

'Office for Education Accreditation,

Pusan National University, Busan,

South Korea

\section{Springer}

\begin{abstract}
In this work, we investigate blowup phenomena for nonlinearly damped viscoelastic equations with logarithmic source effect and time delay in the velocity. Owing to the nonlinear damping term instead of strong or linear dissipation, we cannot apply the concavity method introduced by Levine. Thus, utilizing the energy method, we show that the solutions with not only non-positive initial energy but also some positive initial energy blow up at a finite point in time.
\end{abstract}

MSC: 35L05; 35L70; 35B44

Keywords: Blowup; Viscoelastic equation; Logarithmic source; Time delay; Nonlinear dissipation

\section{Introduction}

We discuss the viscoelastic wave equation with nonlinear damping, logarithmic source, and delay terms

$$
\begin{aligned}
& u_{t t}-\Delta u+k * \Delta u+c_{1}\left|u_{t}(t)\right|^{q-2} u_{t}(t)+c_{2}\left|u_{t}(t-\tau)\right|^{q-2} u_{t}(t-\tau) \\
& \quad=|u|^{p-2} u \ln |u| \quad \text { in } \Omega \times(0, T), \\
& u=0 \quad \text { on } \partial \Omega \times(0, T), \\
& u(0)=u_{0}, \quad u_{t}(0)=u_{1} \quad \text { in } \Omega, \\
& u_{t}(t-\tau)=j_{0}(t-\tau) \quad \text { for } t \in(0, \tau),
\end{aligned}
$$

here $\Omega \subset \mathbb{R}^{n}$ is a bounded domain with smooth boundary $\partial \Omega, k * \Delta u=\int_{0}^{t} k(t-s) \Delta u(s) d s$, the kernel function $k:[0, \infty) \rightarrow(0, \infty)$ is a $C^{1}$-function with

$$
k^{\prime}(t) \leq 0 \quad \text { and } \quad 1-\int_{0}^{\infty} k(s) d s:=k_{l}>0
$$

$\tau>0$ is time delay, the coefficients $c_{1}>0$ and $c_{2} \in \mathbb{R}$ satisfy $0<\left|c_{2}\right|<c_{1}$, and the exponents $q \geq 2$ and $p>2$ are specified later.

(c) The Author(s) 2021. This article is licensed under a Creative Commons Attribution 4.0 International License, which permits use, sharing, adaptation, distribution and reproduction in any medium or format, as long as you give appropriate credit to the original author(s) and the source, provide a link to the Creative Commons licence, and indicate if changes were made. The images or other third party material in this article are included in the article's Creative Commons licence, unless indicated otherwise in a credit line to the material. If material is not included in the article's Creative Commons licence and your intended use is not permitted by statutory regulation or exceeds the permitted use, you will need to obtain permission directly from the copyright holder. To view a copy of this licence, visit http://creativecommons.org/licenses/by/4.0/. 
Many researchers have studied parabolic or hyperbolic equations with logarithmic nonlinearity $[2,4,7,13,15]$. For the physical application of this nonlinearity, we refer to $[1,6]$. In [4], the authors discussed a strongly damped equation,

$$
u_{t t}-\Delta u-\Delta u_{t}=|u|^{p-2} u \ln |u|
$$

with Dirichlet boundary condition. They showed that the solutions with subcritical and critical initial energy blow up in a finite point under suitable conditions. Moreover, they estimated bounds of the blowup time. The authors of [7] proved similar results to those of [4] for the equation with memory. Most work dealing with wave equations with logarithmic nonlinearity is associated with a strongly or linearly damped mechanism, and blowup results are investigated by virtue of the potential well method and Levine's concavity technique [12].

On the other hand, time delay effect arises in many natural phenomena depending not only on the present state but also on some past occurrences. Thus, partial differential equations with time delay have become an active area of research in resent years. For the physical application of the time delay, we refer to $[3,18]$. Recently, Kafini and Messaoudi [8] considered the wave equation with linear damping and delay terms

$$
u_{t t}-\Delta u+c_{1} u_{t}(t)+c_{2} u_{t}(t-\tau)=k|u|^{p-2} u \ln |u|
$$

with Dirichlet boundary condition. They established a blowup result of the solution with negative initial energy by adapting the energy method. While there are many studies on the existence and asymptotic stability of the solutions of wave equations with delay, there are relatively few studies on blowup. We refer to [5, 10, 16, 17, 19] and [9] for stability and blowup of equations with delay, respectively. Motivated by this pioneering work [8], in this article, we study blowup phenomena for the nonlinearly damped viscoelastic wave equation (1.1)-(1.4) with logarithmic source effect and time delay in the velocity. Due to the presence of nonlinear dissipation instead of strong or linear damping terms, we cannot apply the concavity method. Thus, by applying the energy method, we establish a blowup result of solutions with not only non-positive initial energy but also some positive initial energy. And, it is worth to mention that there are few works dealing with viscoelastic wave equations with nonlinear damping and logarithmic source terms.

Here is the outline of this paper. In Sect. 2, we present notations, hypotheses, and auxiliary functions and lemmas. In Sect. 3, we establish a blowup criterion of solutions with not only non-positive initial energy but also some positive initial energy.

\section{Preliminaries}

Throughout this article, $(\cdot, \cdot)$ denotes the scalar product in Hilbert space $L^{2}(\Omega) \cdot\|\cdot\|_{r}$ represents the norm in the space $L^{r}(\Omega)$. Moreover, $\|\cdot\|_{Y}$ denotes the norm of a normed space $Y$. $C>0$ represents a generic constant. If there is no ambiguity, we omit the variables $t$ and $x$.

We let the function $y$ be as in [16]

$$
y(x, \sigma, t)=u_{t}(x, t-\sigma \tau) \quad \text { for }(x, \sigma, t) \in \Omega \times(0,1) \times(0, T) .
$$


Then problem (1.1)-(1.4) reads

$$
\begin{aligned}
& u_{t t}-\Delta u+\int_{0}^{t} k(t-s) \Delta u(s) d s+c_{1}\left|u_{t}\right|^{q-2} u_{t}+c_{2}|y(x, 1, t)|^{q-2} y(x, 1, t) \\
& \quad=|u|^{p-2} u \ln |u| \quad \text { in } \Omega \times(0, T), \\
& \tau y_{t}(x, \sigma, t)+y_{\sigma}(x, \sigma, t)=0 \quad \text { for }(x, \sigma, t) \in \Omega \times(0,1) \times(0, T), \\
& u=0 \quad \text { on } \partial \Omega \times(0, T), \\
& u(0)=u_{0}, \quad u_{t}(0)=u_{1} \quad \text { in } \Omega \\
& y(0)=j_{0}(x,-\sigma \tau):=y_{0} \quad \text { in } \Omega \times(0,1) .
\end{aligned}
$$

By the arguments of $[7,9]$, we can state the well-posedness.

Theorem 2.1 Let $\left(u_{0}, u_{1}, y_{0}\right) \in H_{0}^{1}(\Omega) \times L^{2}(\Omega) \times L^{q}(\Omega \times(0,1)), q \geq 2$, and

$$
2<p<\frac{2(n-1)}{n-2} \quad \text { if } n \geq 3, \quad 2<p<\infty \quad \text { if } n=1,2 .
$$

Then problem (2.1)-(2.5) admits a unique local solution $(u, y)$ with $u \in C\left(0, T ; H_{0}^{1}(\Omega)\right) \cap$ $C^{1}\left(0, T ; L^{2}(\Omega)\right)$ and $y \in L^{\infty}\left(0, T ; L^{q}(\Omega \times(0,1))\right)$.

Our goal is to find a blowup result to problem (2.1)-(2.5). For this, we will often use the embedding

$$
H_{0}^{1}(\Omega) \hookrightarrow L^{r}(\Omega), \quad \text { where } 2 \leq r \leq \bar{r}:= \begin{cases}\infty & \text { if } n=1,2 ; \\ \frac{2 n}{n-2} & \text { if } n \geq 3\end{cases}
$$

and Young's inequality

$$
a b \leq \frac{1}{r} a^{r}+\frac{1}{r^{*}} b^{r^{*}}
$$

where

$$
a, b \geq 0, \quad r, r^{*}>1, \quad \frac{1}{r}+\frac{1}{r^{*}}=1
$$

Also, we need the lemmas below, which are proved by Kafini and Messaoudi [8], to estimate logarithmic nonlinearity.

Lemma 2.1 For $\phi \in L^{p}(\Omega) \cap H_{0}^{1}(\Omega)$, we have

$$
\|\phi\|_{p}^{s} \leq C\left(\|\phi\|_{p}^{p}+\|\nabla \phi\|_{2}^{2}\right) \quad \text { for } 2 \leq s \leq p
$$

Lemma 2.2 For $\phi \in L^{p+1}(\Omega) \cap H_{0}^{1}(\Omega)$ with $\int_{\Omega}|\phi|^{p} \ln |\phi| d x \geq 0$, we have

$$
\left(\int_{\Omega}|\phi|^{p} \ln |\phi| d x\right)^{\frac{s}{p}} \leq C\left(\|\nabla \phi\|_{2}^{2}+\int_{\Omega}|\phi|^{p} \ln |\phi| d x\right) \quad \text { for } 2 \leq s \leq p .
$$


Lemma 2.3 For $\phi \in L^{p}(\Omega) \cap H_{0}^{1}(\Omega)$ with $\int_{\Omega}|\phi|^{p} \ln |\phi| d x \geq 0$, we have

$$
\|\phi\|_{p}^{p} \leq C\left(\|\nabla \phi\|_{2}^{2}+\int_{\Omega}|\phi|^{p} \ln |\phi| d x\right) .
$$

To establish our desired blowup result, we impose the following assumptions:

$\left(H_{n}\right)$ Let $1 \leq n \leq 5$.

$\left(H_{p}\right)$ Let $p$ satisfy

$$
\begin{cases}2<p<\infty & \text { if } n=1,2 \\ 2<p<\min \left\{\frac{2(n-1)}{n-2}, \frac{n+2}{n-2}\right\} & \text { if } n=3,4,5\end{cases}
$$

$\left(H_{q}\right)$ Let $q$ verify

$$
\max \left\{2, \frac{p^{2}+2 p}{p^{2}-2 p+4}\right\}<q<p
$$

$\left(H_{k}\right)$ Let $k$ satisfy

$$
\int_{0}^{\infty} k(s) d s<\frac{p(1-\lambda)-2}{p(1-\lambda)-2+\frac{1}{2 \eta}},
$$

where

$$
0<\lambda<\frac{p-2}{p}, \quad 0<\eta<\frac{p(1-\lambda)}{2} .
$$

From $\left(H_{p}\right)$, there exists $\mu>0$ satisfying

$$
\begin{cases}2<p<p+\mu<\infty & \text { if } n=1,2 \\ 2<p<p+\mu<\frac{2 n}{n-2} & \text { if } n=3,4,5\end{cases}
$$

This implies

$$
H_{0}^{1}(\Omega) \hookrightarrow L^{p+\mu}(\Omega)
$$

Put $D_{0}$ be the embedding constant with

$$
\|\phi\|_{p+\mu} \leq D_{0}\|\nabla \phi\|_{2}, \quad \phi \in H_{0}^{1}(\Omega) .
$$

We let $D=\frac{D_{0}}{\sqrt{k_{l}}}$ and define a continuously differentiable function $K$ as

$$
K(\xi)=-\frac{D^{p+\mu}}{e \mu p} \xi^{p+\mu}+\frac{1}{2} \xi^{2}
$$


and put

$$
\begin{aligned}
& \xi_{K}=\left(\frac{e \mu p}{p+\mu}\right)^{\frac{1}{p+\mu-2}}\left(\frac{1}{D}\right)^{\frac{p+\mu}{p+\mu-2}}, \\
& K_{\max }=\left(\frac{1}{2}-\frac{1}{p+\mu}\right)\left(\frac{e \mu p}{p+\mu}\right)^{\frac{2}{p+\mu-2}}\left(\frac{1}{D}\right)^{\frac{2(p+\mu)}{p+\mu-2}} .
\end{aligned}
$$

Lemma 2.4 For $p>2$ and $\mu>0$, the function $K$ satisfies

(i) $K(0)=0$,

(ii) $\lim _{\xi \rightarrow \infty} K(\xi)=-\infty$,

(iii) $K^{\prime}(\xi)>0$ on $\left(0, \xi_{K}\right), K^{\prime}(\xi)<0$ on $\left(\xi_{K}, \infty\right)$,

(iv) $K$ has the maximum value $K_{\max }$ at $\xi_{K}$.

Proof The results (i) and (ii) are clear. Since

$$
K^{\prime}(\xi)=\xi\left(1-\frac{(p+\mu) D^{p+\mu}}{e \mu p} \xi^{p+\mu-2}\right)
$$

we have $K^{\prime}\left(\xi_{K}\right)=0, K^{\prime}(\xi)>0$ on $\left(0, \xi_{K}\right)$, and $K^{\prime}(\xi)<0$ on $\left(\xi_{K}, \infty\right)$. Thus, $K$ has the maximum value

$$
\begin{aligned}
K\left(\xi_{K}\right) & =-\frac{D^{p+\mu}}{e \mu p} \xi_{K}^{p+\mu}+\frac{1}{2} \xi_{K}^{2} \\
& =\left(\frac{1}{2}-\frac{D^{p+\mu}}{e \mu p} \xi_{K}^{p+\mu-2}\right) \xi_{K}^{2} \\
& =\left(\frac{1}{2}-\frac{1}{p+\mu}\right) \xi_{K}^{2} \\
& =\left(\frac{1}{2}-\frac{1}{p+\mu}\right)\left(\frac{e \mu p}{p+\mu}\right)^{\frac{2}{p+\mu-2}}\left(\frac{1}{D}\right)^{\frac{2(p+\mu)}{p+\mu-2}} .
\end{aligned}
$$

We also need the following auxiliary result in the proof of our main theorem.

Lemma 2.5 For $p>2, \mu>0$, and $0<\lambda<\frac{p-2}{p}$, the $\xi_{K}$ and $K_{\max }$ verify

$$
0<\frac{\xi_{K}^{2}}{2 p(1-\lambda)}\left(\frac{p(1-\lambda)}{2}-1\right)<K_{\max } .
$$

Proof First, we claim

$$
A:=\left(p^{2}-2 p+\mu p+2 \mu\right)-\lambda p(p+\mu-4)>0 .
$$

Indeed, if $p+\mu \leq 4$, it is clear that $A>0$. If $p+\mu>4$,

$$
\min \left\{\frac{p-2}{p}, \frac{p^{2}-2 p+\mu p+2 \mu}{p(p+\mu-4)}\right\}=\frac{p-2}{p} .
$$


This means

$$
0<\lambda<\frac{p-2}{p} \leq \frac{p^{2}-2 p+\mu p+2 \mu}{p(p+\mu-4)} .
$$

So, we also have $A>0$ if $p+\mu>4$. The result (2.18) implies

$$
\begin{aligned}
\frac{1}{2} & -\frac{1}{p+\mu}-\frac{1}{2 p(1-\lambda)}\left(\frac{p(1-\lambda)}{2}-1\right) \\
& =\frac{\left(p^{2}-2 p+\mu p+2 \mu\right)-\lambda p(p+\mu-4)}{4 p(p+\mu)(1-\lambda)}>0,
\end{aligned}
$$

which gives

$$
0<\frac{\xi_{K}^{2}}{2 p(1-\lambda)}\left(\frac{p(1-\lambda)}{2}-1\right)<\left(\frac{1}{2}-\frac{1}{p+\mu}\right) \xi_{K}^{2}=K_{\max } .
$$

\section{Blowup results}

In this part, we search a blowup result of the solution to (2.1)-(2.5) inspired by the ideas in $[8,14]$.

We define the energy to problem (2.1)-(2.5) by

$$
\begin{aligned}
E(t)= & \frac{1}{2}\left\|u_{t}\right\|_{2}^{2}+\frac{1}{2}\left(1-\int_{0}^{t} k(s) d s\right)\|\nabla u\|_{2}^{2}+\frac{1}{2}(k \circ \nabla u)+\frac{1}{p^{2}}\|u\|_{p}^{p} \\
& -\frac{1}{p} \int_{\Omega}|u|^{p} \ln |u| d x+\xi \tau \int_{0}^{1}\|y(\sigma, t)\|_{q}^{q} d \sigma,
\end{aligned}
$$

where

$$
(k \circ \nabla u)(t)=\int_{0}^{t} k(t-s)\|\nabla u(t)-\nabla u(s)\|_{2}^{2} d s
$$

and

$$
\frac{(q-1)\left|c_{2}\right|}{q}<\xi<c_{1}-\frac{\left|c_{2}\right|}{q}
$$

Lemma 3.1 Under the conditions of Theorem 2.1, the equation

$$
\frac{d}{d t} E(t) \leq-\gamma_{1}\left(\left\|u_{t}\right\|_{q}^{q}+\|y(1, t)\|_{q}^{q}\right)-\frac{k(t)}{2}\|\nabla u\|_{2}^{2}+\frac{1}{2}\left(k^{\prime} \circ \nabla u\right) \leq 0
$$

is fulfilled for some $\gamma_{1}>0$.

Proof Taking the scalar product $(2.1)$ by $u_{t}$ in $L^{2}(\Omega)$, we get

$$
\begin{aligned}
& \frac{d}{d t}\left(\frac{1}{2}\left\|u_{t}\right\|_{2}^{2}+\frac{1}{2}\|\nabla u\|_{2}^{2}\right)-\int_{0}^{t} k(t-s)\left(\nabla u(s), \nabla u_{t}(t)\right) d s \\
& \quad=-c_{1}\left\|u_{t}\right\|_{q}^{q}-c_{2} \int_{\Omega} u_{t}(x, t)|y(x, 1, t)|^{q-2} y(x, 1, t) d x+\int_{\Omega} u_{t}|u|^{p-2} u \ln |u| d x .
\end{aligned}
$$


Using the estimate

$$
\int_{\Omega} u_{t}|u|^{p-2} u \ln |u| d x=\frac{1}{p} \frac{d}{d t} \int_{\Omega}|u|^{p} \ln |u| d x-\frac{1}{p^{2}} \frac{d}{d t}\|u\|_{p}^{p}
$$

and

$$
\begin{aligned}
& -\int_{0}^{t} k(t-s)\left(\nabla u(s), \nabla u_{t}(t)\right) d s \\
& =\frac{1}{2} \frac{d}{d t}\left\{(k \circ \nabla u)-\left(\int_{0}^{t} k(s) d s\right)\|\nabla u\|_{2}^{2}\right\}+\frac{k(t)}{2}\|\nabla u\|_{2}^{2}-\frac{1}{2}\left(k^{\prime} \circ \nabla u\right),
\end{aligned}
$$

we get

$$
\begin{aligned}
\frac{d}{d t} E(t)= & -c_{1}\left\|u_{t}(t)\right\|_{q}^{q}-c_{2} \int_{\Omega} u_{t}(x, t)|y(x, 1, t)|^{q-2} y(x, 1, t) d x \\
& -\frac{k(t)}{2}\|\nabla u\|_{2}^{2}+\frac{1}{2}\left(k^{\prime} \circ \nabla u\right)+\frac{\partial}{\partial t}\left(\xi \tau \int_{0}^{1}\|y(\sigma, t)\|_{q}^{q} d \sigma\right) .
\end{aligned}
$$

Using (2.6) with $\frac{q-1}{q}+\frac{1}{q}=1$, we have

$$
-c_{2} \int_{\Omega}|y(x, 1, t)|^{q-2} y(x, 1, t) u_{t}(x, t) d x \leq \frac{\left|c_{2}\right|(q-1)}{q}\|y(1, t)\|_{q}^{q}+\frac{\left|c_{2}\right|}{q}\left\|u_{t}(t)\right\|_{q}^{q} .
$$

From (2.2), we find

$$
\begin{aligned}
\frac{\partial}{\partial t} \int_{0}^{1}\|y(\sigma, t)\|_{q}^{q} d \sigma & =\int_{\Omega} \int_{0}^{1} q|y(x, \sigma, t)|^{q-2} y(x, \sigma, t) y_{t}(x, \sigma, t) d \sigma d x \\
& =-\frac{1}{\tau} \int_{\Omega} \int_{0}^{1} q|y(x, \sigma, t)|^{q-2} y(x, \sigma, t) y_{\sigma}(x, \sigma, t) d \sigma d x \\
& =-\frac{1}{\tau} \int_{\Omega} \int_{0}^{1} \frac{\partial}{\partial \sigma}|y(x, \sigma, t)|^{q} d \sigma d x \\
& =-\frac{1}{\tau}\|y(1, t)\|_{q}^{q}+\frac{1}{\tau}\|y(0, t)\|_{q}^{q} \\
& =-\frac{1}{\tau}\|y(1, t)\|_{q}^{q}+\frac{1}{\tau}\left\|u_{t}(t)\right\|_{q}^{q} .
\end{aligned}
$$

From (3.4), (3.5), and (3.6), one sees

$$
\begin{aligned}
\frac{d}{d t} E(t) \leq & -\left(c_{1}-\frac{\left|c_{2}\right|}{q}-\xi\right)\left\|u_{t}\right\|_{q}^{q}-\left(\xi-\frac{\left|c_{2}\right|(q-1)}{q}\right)\|y(1, t)\|_{q}^{q} \\
& -\frac{k(t)}{2}\|\nabla u\|_{2}^{2}+\frac{1}{2}\left(k^{\prime} \circ \nabla u\right) .
\end{aligned}
$$

Letting

$$
\gamma_{1}=\min \left\{c_{1}-\frac{\left|c_{2}\right|}{q}-\xi, \xi-\frac{\left|c_{2}\right|(q-1)}{q}\right\}
$$

we obtain (3.3) from (3.2). 
Lemma 3.2 Let $(u, y)$ be the solution of (2.1)-(2.5). If the initial datum $\left(u_{0}, y_{0}\right)$ satisfies

$$
E(0)<K_{\max } \text { and } \sqrt{k_{l}}\left\|\nabla u_{0}\right\|_{2} \geq \xi_{K},
$$

there exists $\xi_{1}>\xi_{K}$ such that

$$
\sqrt{k_{l}}\|\nabla u(t)\|_{2} \geq \xi_{1}, \quad t \geq 0
$$

Proof Set

$$
\Omega_{1}=\{x \in \Omega|| u(x, t) \mid \geq 1\} \quad \text { and } \quad \Omega_{2}=\{x \in \Omega|| u(x, t) \mid<1\} .
$$

Using (3.1), (2.11), (2.12), and the relation (Lemma 2.1, [11])

$$
0 \leq \ln a \leq \frac{a^{r}}{e r} \quad \text { for } r>0, a \geq 1,
$$

we derive

$$
\begin{aligned}
E(t) & \geq \frac{1}{2}\left(1-\int_{0}^{t} k(s) d s\right)\|\nabla u\|_{2}^{2}-\frac{1}{p} \int_{\Omega_{1}}|u|^{p} \ln |u| d x-\frac{1}{p} \int_{\Omega_{2}}|u|^{p} \ln |u| d x \\
& \geq \frac{k_{l}}{2}\|\nabla u\|_{2}^{2}-\frac{1}{e \mu p} \int_{\Omega_{1}}|u|^{p+\mu} d x \\
& \geq \frac{k_{l}}{2}\|\nabla u\|_{2}^{2}-\frac{1}{e \mu p}\|u\|_{p+\mu}^{p+\mu} \\
& \geq \frac{k_{l}}{2}\|\nabla u\|_{2}^{2}-\frac{D_{0}^{p+\mu}}{e \mu p}\|\nabla u\|_{2}^{p+\mu} \\
& =K\left(\sqrt{k_{l}}\|\nabla u\|_{2}\right) .
\end{aligned}
$$

Since $E(0)<K_{\max }$, there exists $\xi_{1}>\xi_{K}$ with $K\left(\xi_{1}\right)=E(0)$. From (3.9), we have

$$
K\left(\xi_{1}\right)=E(0) \geq K\left(\sqrt{k_{l}}\left\|\nabla u_{0}\right\|_{2}\right) .
$$

Since $K$ is decreasing on $\left(\xi_{K}, \infty\right),(3.10)$ gives

$$
\sqrt{k_{l}}\left\|\nabla u_{0}\right\|_{2} \geq \xi_{1}
$$

To show (3.8), we use a contradiction. Suppose there exists $t_{1}>0$ such that $\sqrt{k_{l}}\left\|\nabla u\left(t_{1}\right)\right\|_{2}<$ $\xi_{1}$. The continuity of $u$ corresponding to $t$ gives the existence of $t_{0}>0$ with

$$
\xi_{K}<\sqrt{k_{l}}\left\|\nabla u\left(t_{0}\right)\right\|_{2}<\xi_{1} .
$$

From this and (3.9), we get

$$
E\left(t_{0}\right) \geq K\left(\sqrt{k_{l}}\left\|\nabla u\left(t_{0}\right)\right\|_{2}\right)>K\left(\xi_{1}\right)=E(0),
$$

but this contradicts (3.3). 
Now, we are ready to state our main theorem.

Definition 3.1 We say that the solution $(u, y)$ of problem $(2.1)-(2.5)$ blows up in a finite time if there exists a time $T^{*}, 0<T^{*}<\infty$, such that

$$
\lim _{t \rightarrow T^{*}}\|\nabla u(t)\|_{2}=\infty
$$

Theorem 3.1 Let $\left(H_{n}\right),\left(H_{p}\right),\left(H_{q}\right),\left(H_{k}\right)$, and the assumptions of Lemma 3.2 hold. Furthermore, we assume

$$
E(0)<\frac{\xi_{K}^{2}}{2 k_{l} p(1-\lambda)}\left\{k_{l}\left(\frac{p(1-\lambda)}{2}-1\right)-\frac{1}{4 \eta}\left(1-k_{l}\right)\right\} .
$$

Then the solution $(u, y)$ to problem (2.1)-(2.5) blows up after finite time.

Proof Let $\bar{E}$ with

$$
E(0)<\bar{E}<\frac{\xi_{K}^{2}}{2 k_{l} p(1-\lambda)}\left\{k_{l}\left(\frac{p(1-\lambda)}{2}-1\right)-\frac{1}{4 \eta}\left(1-k_{l}\right)\right\} .
$$

From this and (2.17), we see

$$
E(0)<\bar{E}<\frac{\xi_{K}^{2}}{2 p(1-\lambda)}\left(\frac{p(1-\lambda)}{2}-1\right)-\frac{\xi_{K}^{2}\left(1-k_{l}\right)}{8 k_{l} \eta p(1-\lambda)}<K_{\max } .
$$

Define

$$
R(t)=\bar{E}-E(t)
$$

then

$$
R^{\prime}(t)=-E^{\prime}(t) \geq \gamma_{1}\left(\left\|u_{t}\right\|_{q}^{q}+\|y(1, t)\|_{q}^{q}\right)+\frac{k(t)}{2}\|\nabla u\|_{2}^{2}-\frac{1}{2}\left(k^{\prime} \circ \nabla u\right) \geq 0 .
$$

From this, (3.1), (3.12), Lemma 3.2, and the definition of $\xi_{K}$ and $K_{\max }$, we obtain

$$
\begin{aligned}
0 & <R(0) \leq R(t) \leq \bar{E}-\frac{k_{l}}{2}\|\nabla u\|_{2}^{2}+\frac{1}{p} \int_{\Omega}|u|^{p} \ln |u| d x \\
& <K_{\max }-\frac{\xi_{1}^{2}}{2}+\frac{1}{p} \int_{\Omega}|u|^{p} \ln |u| d x \\
& <K_{\max }-\frac{\xi_{K}^{2}}{2}+\frac{1}{p} \int_{\Omega}|u|^{p} \ln |u| d x \\
& =-\frac{D^{p+\mu}}{e \mu p} \xi_{K}^{p+\mu}+\frac{1}{p} \int_{\Omega}|u|^{p} \ln |u| d x \\
& <\frac{1}{p} \int_{\Omega}|u|^{p} \ln |u| d x,
\end{aligned}
$$

which also ensures

$$
\int_{\Omega}|u|^{p} \ln |u| d x>0
$$


Now, we put

$$
L(t)=R^{1-\beta}(t)+\epsilon\left(u(t), u_{t}(t)\right)
$$

where $\epsilon>0$ and

$$
\frac{2(p-q)}{p^{2}(q-1)} \leq \beta \leq \min \left\{\frac{p-q}{p(q-1)}, \frac{p-2}{2 p}\right\}
$$

From (2.1)-(2.5), we get

$$
\begin{aligned}
L^{\prime}(t)= & (1-\beta) R^{-\beta}(t) R^{\prime}(t)+\epsilon\left\|u_{t}\right\|_{2}^{2}-\epsilon\left(1-\int_{0}^{t} k(s) d s\right)\|\nabla u\|_{2}^{2} \\
& +\epsilon \int_{\Omega}|u|^{p} \ln |u| d x+\epsilon \int_{0}^{t} k(t-s)(\nabla u(t), \nabla u(s)-\nabla u(t)) d s \\
& -\epsilon c_{1} \int_{\Omega} u\left|u_{t}\right|^{q-2} u_{t} d x-\epsilon c_{2} \int_{\Omega} u(x, t)|y(x, 1, t)|^{q-2} y(x, 1, t) d x .
\end{aligned}
$$

Using (2.6) with $\frac{q-1}{q}+\frac{1}{q}=1$, we have

$$
c_{1} \int_{\Omega} u\left|u_{t}\right|^{q-2} u_{t} d x \leq \frac{c_{1}(q-1)}{q} \chi^{-\frac{q}{q-1}}\left\|u_{t}\right\|_{q}^{q}+\frac{c_{1} \chi^{q}}{q}\|u\|_{q}^{q}
$$

and

$$
c_{2} \int_{\Omega} u(x, t)|y(x, 1, t)|^{q-2} y(x, 1, t) d x \leq \frac{\left|c_{2}\right| \chi^{q}}{q}\|u\|_{q}^{q}+\frac{\left|c_{2}\right|(q-1)}{q} \chi^{-\frac{q}{q-1}}\|y(1, t)\|_{q}^{q}
$$

for any $\chi>0$. From these and the relation $0<\left|c_{2}\right|<c_{1}$, we get

$$
\begin{aligned}
& c_{1} \int_{\Omega} u\left|u_{t}\right|^{q-2} u_{t} d x+c_{2} \int_{\Omega} u(x, t)|y(x, 1, t)|^{q-2} y(x, 1, t) d x \\
& \quad \leq \frac{c_{1}(q-1)}{q} \chi^{-\frac{q}{q-1}\left(\left\|u_{t}\right\|_{q}^{q}+\|y(1, t)\|_{q}^{q}\right)+\frac{\left(c_{1}+\left|c_{2}\right|\right) \chi^{q}}{q}\|u\|_{q}^{q} .}
\end{aligned}
$$

Taking $\chi=\left(\theta R^{-\beta}(t)\right)^{-\frac{q-1}{q}}, \theta>0$, and applying (3.14), we derive

$$
\begin{aligned}
& c_{1} \int_{\Omega} u\left|u_{t}\right|^{q-2} u_{t} d x+c_{2} \int_{\Omega} u|y(1, t)|^{q-2} y(1, t) d x \\
& \quad \leq \frac{c_{1}(q-1) \theta}{q}(R(t))^{-\beta}\left(\left\|u_{t}\right\|_{q}^{q}+\|y(1, t)\|_{q}^{q}\right)+\frac{c_{1}+\left|c_{2}\right|}{q \theta^{q-1}}(R(t))^{\beta(q-1)}\|u\|_{q}^{q} \\
& \quad \leq \frac{c_{1}(q-1) \theta}{q \gamma_{1}}(R(t))^{-\beta} R^{\prime}(t)+\frac{C}{q \theta^{q-1}}(R(t))^{\beta(q-1)}\|u\|_{p}^{q} .
\end{aligned}
$$

Using (3.16), (3.15), Lemma 2.3, and (2.6) with $\frac{q}{p}+\frac{p-q}{p}=1$, we find

$$
\begin{aligned}
& (R(t))^{\beta(q-1)}\|u\|_{p}^{q} \\
& \quad \leq C\left(\int_{\Omega}|u|^{p} \ln |u| d x\right)^{\beta(q-1)}\left(\int_{\Omega}|u|^{p} \ln |u| d x+\|\nabla u\|_{2}^{2}\right)^{\frac{q}{p}}
\end{aligned}
$$




$$
\begin{aligned}
& \leq C\left(\int_{\Omega}|u|^{p} \ln |u| d x\right)^{\beta(q-1)}\left\{\left(\int_{\Omega}|u|^{p} \ln |u| d x\right)^{\frac{q}{p}}+\|\nabla u\|_{2}^{\frac{2 q}{p}}\right\} \\
& \leq C\left(\int_{\Omega}|u|^{p} \ln |u| d x\right)^{\beta(q-1)+\frac{q}{p}}+C\left(\int_{\Omega}|u|^{p} \ln |u| d x\right)^{\beta(q-1)}\|\nabla u\|_{2}^{\frac{2 q}{p}} \\
& \leq C\left(\int_{\Omega}|u|^{p} \ln |u| d x\right)^{\frac{\beta p(q-1)+q}{p}}+C\left(\int_{\Omega}|u|^{p} \ln |u| d x\right)^{\frac{\beta(q-1) p}{p-q}}+\|\nabla u\|_{2}^{2} .
\end{aligned}
$$

From (3.18), we see $0<\beta \leq \frac{p-q}{p(q-1)}$, which gives

$$
2 \leq \beta p(q-1)+q \leq p
$$

Thanks to $\left(H_{p}\right)$, we also note the solution $u$ to $(2.1)-(2.5)$ belongs to $L^{p+1}(\Omega)$. So, we can apply Lemma 2.2 to get

$$
\left(\int_{\Omega}|u|^{p} \ln |u| d x\right)^{\frac{\beta p(q-1)+q}{p}} \leq C\left(\|\nabla u\|_{2}^{2}+\int_{\Omega}|u|^{p} \ln |u| d x\right) .
$$

Similarly, from (3.18), we see $\frac{2(p-q)}{p^{2}(q-1)} \leq \beta \leq \frac{p-q}{p(q-1)}$, which implies

$$
2 \leq \frac{\beta(q-1) p^{2}}{p-q} \leq p
$$

So, we have

$$
\left(\int_{\Omega}|u|^{p} \ln |u| d x\right)^{\frac{\beta(q-1) p}{p-q}} \leq C\left(\|\nabla u\|_{2}^{2}+\int_{\Omega}|u|^{p} \ln |u| d x\right) .
$$

Inserting (3.22) and (3.23) to (3.21), we obtain

$$
(R(t))^{\beta(q-1)}\|u\|_{p}^{q} \leq C\left(\|\nabla u\|_{2}^{2}+\int_{\Omega}|u|^{p} \ln |u| d x\right)
$$

From this and (3.20),

$$
\begin{aligned}
& c_{1} \int_{\Omega}\left|u_{t}\right|^{q-2} u_{t} d x+c_{2} \int_{\Omega} u|y(1, t)|^{q-2} y(1, t) d x \\
& \quad \leq \frac{c_{1}(q-1) \theta}{q \gamma_{1}}(R(t))^{-\beta} R^{\prime}(t)+\frac{C}{q \theta^{q-1}}\left(\int_{\Omega}|u|^{p} \ln |u| d x+\|\nabla u\|_{2}^{2}\right) .
\end{aligned}
$$

Applying this and the estimate

$$
\int_{0}^{t} k(t-s)(\nabla u(t), \nabla u(s)-\nabla u(t)) d s \geq-\eta(k \circ \nabla u)-\frac{1}{4 \eta}\left(\int_{0}^{t} k(s) d s\right)\|\nabla u\|_{2}^{2}
$$

to (3.19), we get

$$
L^{\prime}(t) \geq\left((1-\beta)-\frac{\epsilon c_{1}(q-1) \theta}{q \gamma_{1}}\right) R^{-\beta}(t) R^{\prime}(t)+\epsilon\left\|u_{t}\right\|_{2}^{2}
$$




$$
\begin{aligned}
& +\epsilon \int_{\Omega}|u|^{p} \ln |u| d x-\epsilon \eta(k \circ \nabla u)-\frac{\epsilon C}{q \theta^{q-1}} \int_{\Omega}|u|^{p} \ln |u| d x \\
& -\epsilon\left(1-\int_{0}^{t} k(s) d s+\frac{1}{4 \eta} \int_{0}^{t} k(s) d s+\frac{C}{q \theta^{q-1}}\right)\|\nabla u\|_{2}^{2} .
\end{aligned}
$$

Subtracting and adding the term $\epsilon \lambda \int_{\Omega}|u|^{p} \ln |u| d x$, where $\lambda$ is given in (2.9), and using (3.1) and (3.13), we get

$$
\begin{aligned}
L^{\prime}(t) \geq & \left((1-\beta)-\frac{\epsilon c_{1}(q-1) \theta}{q \gamma_{1}}\right) R^{-\beta}(t) R^{\prime}(t)+\epsilon\left(1+\frac{p(1-\lambda)}{2}\right)\left\|u_{t}\right\|_{2}^{2} \\
& +\epsilon\left(\lambda-\frac{C}{q \theta^{q-1}}\right) \int_{\Omega}|u|^{p} \ln |u| d x+\frac{\epsilon(1-\lambda)}{p}\|u\|_{p}^{p}+\epsilon p(1-\lambda) R(t) \\
& +\epsilon \xi \tau p(1-\lambda) \int_{0}^{1}\|y(\sigma, t)\|_{q}^{q} d \sigma+\epsilon\left(\frac{p(1-\lambda)}{2}-\eta\right)(k \circ \nabla u)-\epsilon p(1-\lambda) \bar{E} \\
& +\frac{\epsilon}{2}\left\{k_{l}\left(\frac{p(1-\lambda)}{2}-1\right)-\frac{1}{4 \eta}\left(1-k_{l}\right)-\frac{C}{q \theta^{q-1}}\right\}\|\nabla u\|_{2}^{2} \\
& +\frac{\epsilon}{2}\left\{k_{l}\left(\frac{p(1-\lambda)}{2}-1\right)-\frac{1}{4 \eta}\left(1-k_{l}\right)-\frac{C}{q \theta^{q-1}}\right\}\|\nabla u\|_{2}^{2} .
\end{aligned}
$$

By $\left(H_{k}\right)$, we note

$$
\frac{p(1-\lambda)}{2}-\eta>0
$$

and

$$
k_{l}\left(\frac{p(1-\lambda)}{2}-1\right)-\frac{1}{4 \eta}\left(1-k_{l}\right)>0 .
$$

Firstly, we take $\theta>0$ appropriately large to guarantee

$$
\lambda-\frac{C}{q \theta^{q-1}}>0
$$

and

$$
k_{l}\left(\frac{p(1-\lambda)}{2}-1\right)-\frac{1}{4 \eta}\left(1-k_{l}\right)-\frac{C}{q \theta^{q-1}}>0 .
$$

Next, we claim

$$
-\epsilon p(1-\lambda) \bar{E}+\frac{\epsilon}{2}\left\{k_{l}\left(\frac{p(1-\lambda)}{2}-1\right)-\frac{1}{4 \eta}\left(1-k_{l}\right)-\frac{C}{q \theta^{q-1}}\right\}\|\nabla u\|_{2}^{2}>0 .
$$

Indeed, it is seen from Lemma 3.2 that

$$
\begin{array}{r}
-p(1-\lambda) \bar{E}+\frac{1}{2}\left\{k_{l}\left(\frac{p(1-\lambda)}{2}-1\right)-\frac{1}{4 \eta}\left(1-k_{l}\right)-\frac{C}{q \theta^{q-1}}\right\}\|\nabla u\|_{2}^{2} \\
\geq-p(1-\lambda) \bar{E}+\frac{\xi_{1}^{2}}{2 k_{l}}\left\{k_{l}\left(\frac{p(1-\lambda)}{2}-1\right)-\frac{1}{4 \eta}\left(1-k_{l}\right)-\frac{C}{q \theta^{q-1}}\right\} \\
>-p(1-\lambda) \bar{E}+\frac{\xi_{K}^{2}}{2 k_{l}}\left\{k_{l}\left(\frac{p(1-\lambda)}{2}-1\right)-\frac{1}{4 \eta}\left(1-k_{l}\right)-\frac{C}{q \theta^{q-1}}\right\} .
\end{array}
$$


From (3.11), we have

$$
-p(1-\lambda) \bar{E}+\frac{\xi_{K}^{2}}{2 k_{l}}\left\{k_{l}\left(\frac{p(1-\lambda)}{2}-1\right)-\frac{1}{4 \eta}\left(1-k_{l}\right)\right\}>0 .
$$

Thus, we can fix $\theta>0$ suitably large again to get

$$
-p(1-\lambda) \bar{E}+\frac{\xi_{K}^{2}}{2 k_{l}}\left\{k_{l}\left(\frac{p(1-\lambda)}{2}-1\right)-\frac{1}{4 \eta}\left(1-k_{l}\right)\right\}-\frac{C}{2 k_{l} q \theta^{q-1}} \xi_{K}^{2}>0 .
$$

This and (3.27) imply (3.26).

Finally, we pick $\epsilon>0$ suitably small to have

$$
(1-\beta)-\frac{\epsilon c_{1}(q-1) \theta}{q \gamma_{1}}>0
$$

and

$$
L(0)=R^{1-\beta}(0)+\epsilon\left(u_{0}, u_{1}\right)>0 .
$$

Therefore, from (3.25) we arrive at

$$
L^{\prime}(t) \geq C\left(\left\|u_{t}\right\|_{2}^{2}+\|u\|_{p}^{p}+R(t)+\int_{\Omega}|u|^{p} \ln |u| d x+\|\nabla u\|_{2}^{2}\right) .
$$

Next, from (3.17), we know

$$
L^{\frac{1}{1-\beta}}(t) \leq C\left(R(t)+\left|\left(u, u_{t}\right)\right|^{\frac{1}{1-\beta}}\right) .
$$

Using (2.6) with $\frac{1}{2(1-\beta)}+\frac{1-2 \beta}{2(1-\beta)}=1$, noting $2 \leq \frac{2}{1-2 \beta} \leq p$ from (3.18), and applying Lemma 2.1, we deduce

$$
\begin{aligned}
\left|\left(u, u_{t}\right)\right|^{\frac{1}{1-\beta}} & \leq\|u\|_{2}^{\frac{1}{1-\beta}}\left\|u_{t}\right\|_{2}^{\frac{1}{1-\beta}} \\
& \leq C\|u\|_{p}^{\frac{1}{1-\beta}}\left\|u_{t}\right\|_{2}^{\frac{1}{1-\beta}} \\
& \leq C\left(\left\|u_{t}\right\|_{2}^{2}+\|u\|_{p}^{\frac{2}{1-2 \beta}}\right) \\
& \leq C\left(\left\|u_{t}\right\|_{2}^{2}+\|u\|_{p}^{p}+\|\nabla u\|_{2}^{2}\right) .
\end{aligned}
$$

Combining (3.29), (3.30) and noting (3.16), we derive

$$
\begin{aligned}
L^{\frac{1}{1-\beta}}(t) & \leq C\left(R(t)+\left\|u_{t}\right\|_{2}^{2}+\|u\|_{p}^{p}+\|\nabla u\|_{2}^{2}\right) \\
& \leq C\left(R(t)+\left\|u_{t}\right\|_{2}^{2}+\|u\|_{p}^{p}+\|\nabla u\|_{2}^{2}+\int_{\Omega}|u|^{p} \ln |u| d x\right) .
\end{aligned}
$$

From this and (3.29), we conclude

$$
L^{\prime}(t) \geq C L^{\frac{1}{1-\beta}}(t), \quad t \geq 0
$$

which shows that the solution $u$ blows up after finite time $T^{*} \leq \frac{1-\beta}{C \beta L^{\frac{\beta}{1-\beta}}(0)}$. 


\section{Conclusion}

In this paper, we considered a viscoelastic wave equation with nonlinear damping and time delay terms and logarithmic source effect. Under the conditions $\left(H_{n}\right),\left(H_{p}\right),\left(H_{q}\right)$, and $\left(H_{k}\right)$, we showed that the solutions with not only a non-positive initial energy but also some positive initial energy blow up after a finite time by utilizing the energy method.

\section{Acknowledgements}

The author is grateful to the anonymous referees for their careful reading and important comments.

\section{Funding}

This work was supported by Basic Science Research Program through the National Research Foundation of Korea(NRF) funded by the Ministry of Education (2020R111A3066250).

\section{Availability of data and materials}

Not applicable.

\section{Competing interests}

The author declares that they have no competing interests.

\section{Authors' contributions}

The author read and approved the final manuscript.

\section{Publisher's Note}

Springer Nature remains neutral with regard to jurisdictional claims in published maps and institutional affiliations.

Received: 15 February 2021 Accepted: 18 June 2021 Published online: 30 June 2021

\section{References}

1. Barrow, J., Parsons, P.: Inflationary models with logarithmic potentials. Phys. Rev. D 52, 5576-5587 (1995)

2. Cao, Y., Liu, C.: Initial boundary value problem for a mixed pseudo-parabolic p-Laplacian type equation with logarithmic nonlinearity. Electron. J. Differ. Equ. 2018, Article ID 116 (2018)

3. Datko, R.: Not all feedback stabilized hyperbolic systems are robust with respect to small time delays in their feedbacks. SIAM J. Control Optim. 26, 697-713 (1988)

4. Di, H., Shang, Y., Song, Z.: Initial boundary value problems for a class of strongly damped semilinear wave equations with logarithmic nonlinearity. Nonlinear Anal., Real World Appl. 51, 102968 (2020)

5. Feng, B.: Global well-posedness and stability for a viscoelastic plate equation with a time delay. Math. Probl. Eng. 2015, Article ID 585021 (2015)

6. Gorka, P.: Logarithmic Klein-Gordon equation. Acta Phys. Pol. B 40, 59-66 (2009)

7. Ha, T.G., Park, S.H.: Blow-up phenomena for a viscoelastic wave equation with strong damping and logarithmic nonlinearity. Adv. Differ. Equ. 2020, Article ID 235 (2020)

8. Kafini, M., Messaoudi, S.: Local existence and blow up of solutions to a logarithmic nonlinear wave equation with delay. Appl. Anal. 99, 530-547 (2020)

9. Kafini, M., Messaoudi, S.A., Nicaise, S.: A blow-up result in a nonlinear abstract evolution system with delay. Nonlinear Differ. Equ. Appl. 23, Article ID 13 (2016)

10. Kirane, M., Said-Houari, B.: Existence and asymptotic stability of a viscoelastic wave equation with a delay. Z. Angew. Math. Phys. 62, 1065-1082 (2011)

11. Le, C.N., Le, X.T.: Global solution and blow-up for a class of $p$-Laplacian evolution equations with logarithmic nonlinearity. Acta Appl. Math. 151, 149-169 (2017)

12. Levine, H.A.: Instability and nonexistence of global solutions of nonlinear wave equation of the form $P u_{t t}=A u+F(u)$. Trans. Am. Math. Soc. 192, 1-21 (1974)

13. Liao, M., Li, Q.: A class of fourth-order parabolic equations with logarithmic nonlinearity. Taiwan. J. Math. 24, 975-1003 (2020)

14. Messaoudi, S.A.: Blow-up of positive-initial-energy solutions of a nonlinear viscoelastic hyperbolic equation. J. Math. Anal. Appl. 320, 902-915 (2006)

15. Nhan, L.C., Truong, L.X.: Global solution and blow-up for a class of pseudo p-Laplacian evolution equations with logarithmic nonlinearity. Comput. Math. Appl. 73, 2076-2091 (2017)

16. Nicaise, S., Pignotti, C.: Stability and instability results of the wave equation with a delay term in the boundary or internal feedbacks. SIAM J. Control Optim. 45, 1561-1585 (2006)

17. Park, S.H.: Long-time dynamics of a von Karman equation with time delay. Appl. Math. Lett. 75, $128-134$ (2018)

18. Suh, I.H., Bien, Z.: Use of time delay action in the controller design. IEEE Trans. Autom. Control 25, 600-603 (1980)

19. Yang, Z.: Existence and energy decay of solutions for the Euler-Bernoulli viscoelastic equation with a delay. Z. Angew. Math. Phys. 66, 727-745 (2015) 